\title{
Results of Single-Staged Posterior Decompression and Circumferential Fusion Using a Transpedicular Approach to Correct a Kyphotic Deformity due to Thoracolumbar Spinal Tuberculosis
}

\author{
Shaival Dalal, Jayprakash Modi, Shardul Soman, Harshil Patel, Shrikant Dhanani \\ Department of Orthopaedics, B.J Medical College and Civil Hospital, Ahmedabad, Gujarat, India
}

\begin{abstract}
Study Design: This is a prospective study.
Purpose: The aim of this study was to investigate the results of single-staged posterior decompression and circumferential fusion using a transpedicular approach to correct a kyphotic deformity due to thoracolumbar spinal tuberculosis.

Overview of Literature: Surgical management is frequently an imperative choice to achieve spinal decompression and deformity correction due to tuberculosis to relieve pain, improve neurology, and reconstruct the spine stability. Since the time anterior radical debridement and noninstrumented fusion was described, it has become apparent that even anterior debridement and bone grafting was often unsatisfactory in correcting or preventing the progression of kyphosis deformity. With the advent of modern segmental spinal instrumentation systems, isolated posterior instrumentation; combined anterior and posterior fusion; and single-staged posterior decompression and circumferential fusion have been described by many authors for correcting angular deformity and stabilizing the spine; however, there is a lack of consensus regarding the most effective means of correcting the deformity due to thoracolumbar spinal tuberculosis.

Methods: This is a prospective study of 20 patients with thoracolumbar spinal tuberculosis who underwent surgery at our institute. Results: Twenty patients who were started on antituberculosis treatment underwent surgery using a single-staged posterior approach involving fixation, decompression, and kyphosis correction. Preoperatively, all patients had varying degrees of neurological deficit and a $27.45^{\circ}$ average kyphotic angle, which improved. At the 1 -year follow-up, correction was maintained at $6.9^{\circ}$, and $55 \%$ of patients showed neurological improvement. None of the patients experienced neurological deterioration. Two patients with lumbar spine tuberculosis underwent revision surgery because of nonunion.

Conclusions: The procedure of posterior decompression, fixation, and circumferential fusion using a transpedicular approach performed for thoracolumbar spinal tuberculosis is effective, safe, and excellent in correcting and maintaining kyphosis.
\end{abstract}

Keywords: Spinal tuberculosis; Decompression; Fusion; Kyphosis

Received Jan 3, 2016; Revised Apr 19, 2016; Accepted May 17, 2016

Corresponding author: Shaival Dalal

B.J Medical College and Civil Hospital, Ahmedabad, Gujarat 380016, India

Tel: +91-9904915211, E-mail: shaival_dalal@yahoo.co.in 


\section{Introduction}

Spinal tuberculosis (TB) is a destructive form of TB. It accounts for approximately half of all cases of musculoskeletal TB. The incidence of spinal TB is increasing in developing countries. Gross destruction of the vertebral bodies and discs by TB often leads to kyphotic deformity $[1,2]$. Though many patients can be cured by chemotherapy, surgery is often necessary for spinal decompression and deformity correction. Previous studies showed that surgical treatment is an important strategy for the treatment of spinal TB $[3,4]$.

In 1934, Ito et al. [5] first described the anterior debridement approach for spinal TB; however, this approach may reduce the biomechanical stability of the spine, and it is common to observe residual kyphosis at the end of treatment [6]. Thus, anterior debridement combined with posterior fusion and fixation was developed, which helped arrest the disease early and prevented the progression of kyphosis as well as corrected it. However, the combined procedures were associated with longer surgeries, greater blood loss, more postoperative complications, and a longer hospital stay. The purpose of the present study is to investigate the efficacy and safety of a one-stage posterior transpedicular approach for achieving circumferential fusion.

In recent years, global TB incidence has increased because of population migration, increase in the number of patients with human immunodeficiency virus (HIV) infection, and emergence of antibiotic-resistant strains, among other reasons $[7,8]$. India has the highest burden of TB. The World Health Organization (WHO) statistics for 2014 give an estimated incidence figure of 2.2 million cases of TB for India out of a global incidence of 9 million [9]. In India, TB is not restricted to immunocompromised patients [9]. Spinal TB leads to increased pressure on the spine and therefore, causes neurological injury. The chief aim of surgical treatment is focal clearance of $\mathrm{TB}$, relief of spinal nerve compression, reconstruction of spinal stability, and correction of the spinal deformity. This study reviewed patients with thoracolumbar spinal TB following one-stage fixation, debridement, anterior reconstruction, and circumferential fusion using a single posterior approach only.

\section{Materials and Methods}

The study design of this study is a prospective case series. We have included patients whose thoracolumbar spine was affected. Patients who had levels affected in areas other than the thoracolumbar spine, those who were immunocompromised or HIV positive, or who have undergone any previous surgical interventions for the spine were excluded from our study. Twenty patients who presented with signs and symptoms of vertebral osteomyelitis of the thoracolumbar region were assessed using X-rays, magnetic resonance imaging, erythrocyte sedimentation rate (ESR), and C-reactive protein (CRP) levels. On preoperative assessment, clinical assessment was performed using the visual analogue scale (VAS), neurological assessment was performed using the American Spinal Injury Association (ASIA) scale, and radiological assessment was performed using anterior-posterior and lateral X-rays and sagittal alignment by the measurement of the Cobb angle. We divided the patients in thoracic (T1-T10), thoracolumbar (T11-L2), and lumbar (L3-L5) groups and classified the patients on the basis of the Gulhane Military Medical Academy (GATA) classification developed by Oguz et al. [10]. The patients also underwent posterior transpedicular approach surgery. The details of the surgical technique have been described in the following section. Intraoperative neuromonitoring was not used. Operative time and intraoperative blood loss were recorded. All patients were confirmed to have TB by histopathology testing, sensitivity was detected by a TB gene expert [11], and culture and sensitivity of the pathological specimens were sent preoperatively. After pathological diagnosis, all patients were started on medical management of TB as per Revised National Tuberculosis Control Program (RNTCP) guidelines issued by the government (Table 1) [12,13]; nine patients were already on medical management to treat TB. Patients were observed at intervals of 3 months

Table 1. Revised National Tuberculosis Control Program guidelines [11,12]

\begin{tabular}{lll} 
Category & \multicolumn{1}{c}{ Type } & Regimen \\
\hline I & All "new" pulmonary, extrapulmonary and other TB patients & 2(HRZE)3+4(HR)3 \\
\hline
\end{tabular}

TB, tuberculosis; $H$, isoniazid; $R$, rifampicin; $Z$, pyrizinamide; $E$, etambutol. 
postoperatively for clinical assessment in terms of neurological improvement and VAS, laboratory assessment in terms of ESR and CRP, radiological assessment in terms of the Cobb angle, and assessment of fusion using Lenke's classification [14]. Data were analyzed using the SPSS ver. 13.0 statistical software package (SPSS Inc., Chicago, IL, USA). The paired $t$-test was used to examine the statistical significance between the two groups. All test results were considered significant at $p<0.05$.

\section{Surgical technique}

All patients were operated under general anesthesia while in the prone position. Through a posterior midline approach, the lamina, facet joints, and transverse processes were exposed. Exposing the vertebral laminae of involved segments, the posterior pedicle screws were installed. Laminectomy, according to the extent of cord compression, was performed before debridement of the affected intervertebral discs and vertebrae. Facetectomy and pediculectomy were also performed at appropriate levels. To obtain a broader view, one spinal nerve on the focal side may have been sacrificed in the thoracic levels and abscesses were evacuated. Following completion of the debridement, the deformity was corrected by installing permanent rods with compression maneuvers under vision. Anterior reconstruction was achieved using an appropriate-sized cage, and autograft and posterior interbody grafts were used after ensuring that the spinal cord was not compressed.

\section{Results}

Patients aged 7-80 years were included in the study, and the average age was 37.7 years. Of the 20 patients, 8 (40\%), $4(20 \%), 4(20 \%)$, and $4(20 \%)$ were in $7-30,31-45$, $46-60$, and $>60$ years range, respectively. In our study, 8 patients (40\%) were females and $12(60 \%)$ were males. There were 8 patients in the thoracic (T1-T10) category, 7 patients in thoracolumbar (T11-L2) category, and 5 patients in the lumbar (L3-L5) category (Table 2). All patients belonged to type III according to Oguz classification [10]. No worsening of neurology was observed postoperatively or at follow-up. Better results in the ASIA scale were

Table 2. Demographic and operative data

\begin{tabular}{lrrr} 
Demographic data & Minimum & Maximum & Average \\
\hline Age (yr) & 7 & 80 & 37.7 \\
\hline Operative time (min) & 140 & 210 & 165 \\
\hline Blood loss (mL) & 300 & 650 & 450 \\
Mean follow-up (mo) & 7 & 13 & 10 \\
\hline Involved levels (patients) & & - & 8 \\
T1-T10 & - & - & 7 \\
\hline T11-L2 & - & - & 5 \\
\hline L3-L5 & - & & \\
\hline
\end{tabular}

Table 3. Table matrix showing preoperative and postoperative ASIA scores

\begin{tabular}{lccccc} 
ASIA scale & \multicolumn{3}{c}{ Final follow-up } \\
\cline { 2 - 6 } Preoperative & A & B & C & 0 & D \\
A & 0 & 0 & 0 & 1 & 0 \\
B & 0 & 1 & 0 & 4 & 4 \\
C & 0 & 0 & 0 & 0 & 1 \\
D & 0 & 0 & 0 & 0 & 8 \\
\hline E & 0 & 0 & 0 & 1 \\
\hline
\end{tabular}

ASIA, American Spinal Injury Association. 
Table 4. Comparison of various clinical, radiological and laboratory parameters preoperatively and at final follow-up

\begin{tabular}{lccc} 
Variable (mean) & Preoperative & Final follow-up & $p$-value \\
\hline Visual analogue scale & 6.9 & 2.5 & $<0.05$ \\
\hline Cobb's angle $\left({ }^{\circ}\right)$ & 27.5 & 6.6 & $<0.05$ \\
\hline Erythrocyte sedimentation rate $(\mathrm{mm} / \mathrm{hr})$ & 45.2 & 14.9 & $<0.05$ \\
\hline C-reactive protein & 15 & 1.8 & $<0.05$ \\
\hline
\end{tabular}

recorded in the patients who underwent surgery (Table 3 ). The ASIA scale improved in 11 of the 20 patients, while nine patients had the same scores postoperatively, and two patients who were diagnosed with nonunion showed no worsening in neurology. In one patient who presented with a pathological fracture (ASIA type B), there was no improvement in neurology.

Bony fusion was achieved in $90 \%$ of the patients. Fusion was assessed according to the Lenke classification of X-ray fusion [13]. The Cobb angle improved from an average of $27.5^{\circ}\left(10^{\circ}-85^{\circ}\right)$ preoperatively to a postoperative angle of $6.25^{\circ}\left(0^{\circ}-25^{\circ}\right)$ and a mean $6.95^{\circ}$ at the final follow-up
(Figs. 1-3).

Patients showed improvement from a mean VAS of 6.9 (6-8) to 4.75 (3-6) and 2.55 (1-4) postoperatively and at the 12-month follow-up, respectively (Table 3 ). In our study, preoperative markers of inflammation were raised and then returned to normal at the final follow-up (Table 4).

\section{Discussion}

Spinal TB prevails in many parts of the world and is a severe orthopedic problem [15], usually presenting with kyphosis, neurological deficit, and even paraplegia [16,17].
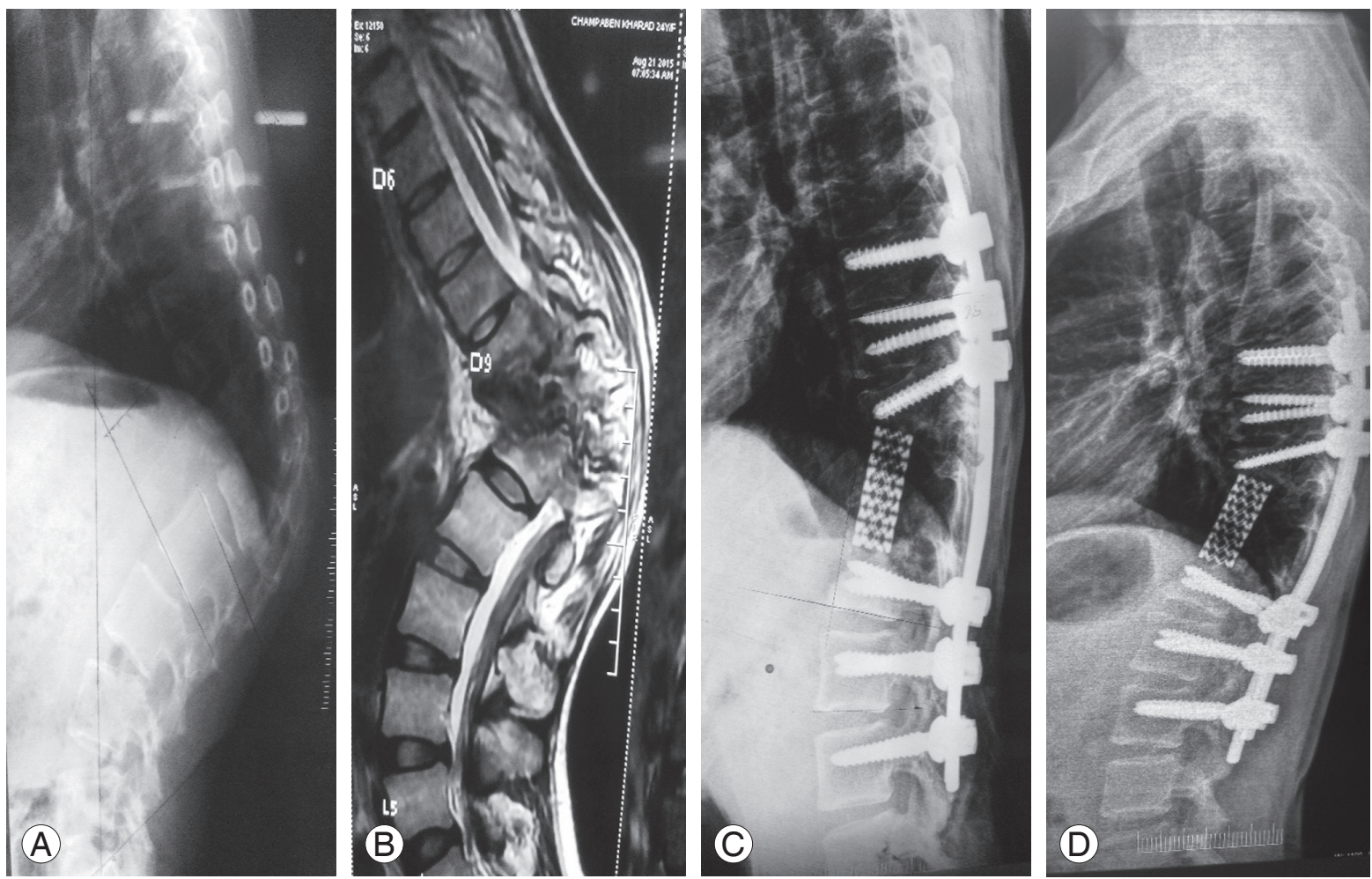

Fig. 1. A 24-year-old female with D9-L1 spinal tuberculosis with American Spinal Injury Association (ASIA) B classification and an $85^{\circ}$ Cobb angle preoperatively underwent surgery using a single-staged transpedicular approach. The postoperative Cobb angle was $25^{\circ}$, and neurology remained the same (ASIA B). At the 12-month follow-up, the Cobb angle was $30^{\circ}$ with no worsening of neurology. (A) Preoperative X-ray. (B) Preoperative magnetic resonance imaging. (C) Postoperative X-ray. (D) Follow-up X-ray. 

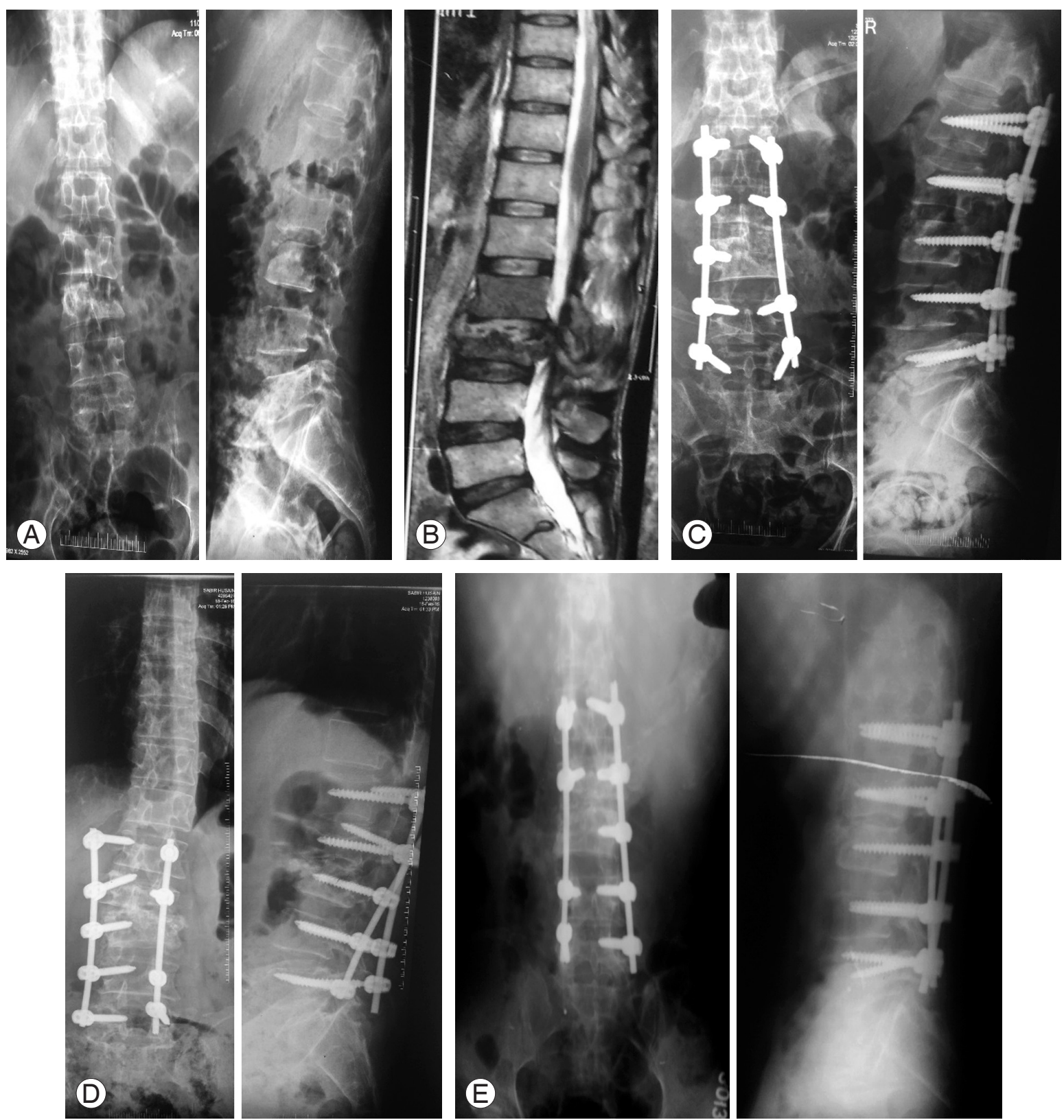

Fig. 2. A 48-year-old male with $\mathrm{L} 2-\mathrm{L} 3$ spinal tuberculosis with American Spinal Injury Association (ASIA) D classification and a $15^{\circ}$ Cobb angle preoperatively underwent surgery using the single-staged transpedicular approach. Immediatelyafter surgery, the Cobb angle was $5^{\circ}$ with no worsening of neurology. At the 6-month follow-up, nonunion (Lenke grade 3) was observed with implant backout. The Cobb angle was $10^{\circ}$, and neurology improved to ASIA E. Revision fixation and bone grafting was performed. The final 12-month follow-up showed a $3^{\circ} \mathrm{Cobb}$ angle with no worsening of neurology. (A) Preoperative $X$-ray. (B) Preoperative magnetic resonance imaging. (C) Postoperative X-ray. (D) Follow-up X-ray. (E) Post revision X-ray.

Thoracolumbar lesions, the junctional region of thoracic and lumbar lesions, have the highest propensity to develop morphological changes likely to predispose to deformity progression in patients with spinal TB [6]. Early therapy has become important for the treatment of thoracolumbar spinal TB. Although anti-TB chemotherapy effectively inactivates TB, vertebral collapse may continue. Kyphosis requiring extensive reconstructive procedures is a common complication of spinal TB in patients treated with chemotherapy alone $[18,19]$. Surgery for TB spondylitis is generally considered to be an adjuvant of effective chemotherapy. Indications for surgery are neurological deficit, spinal instability, severe and/or progressive kyphosis, no response to chemotherapy, and nondiagnostic and large 

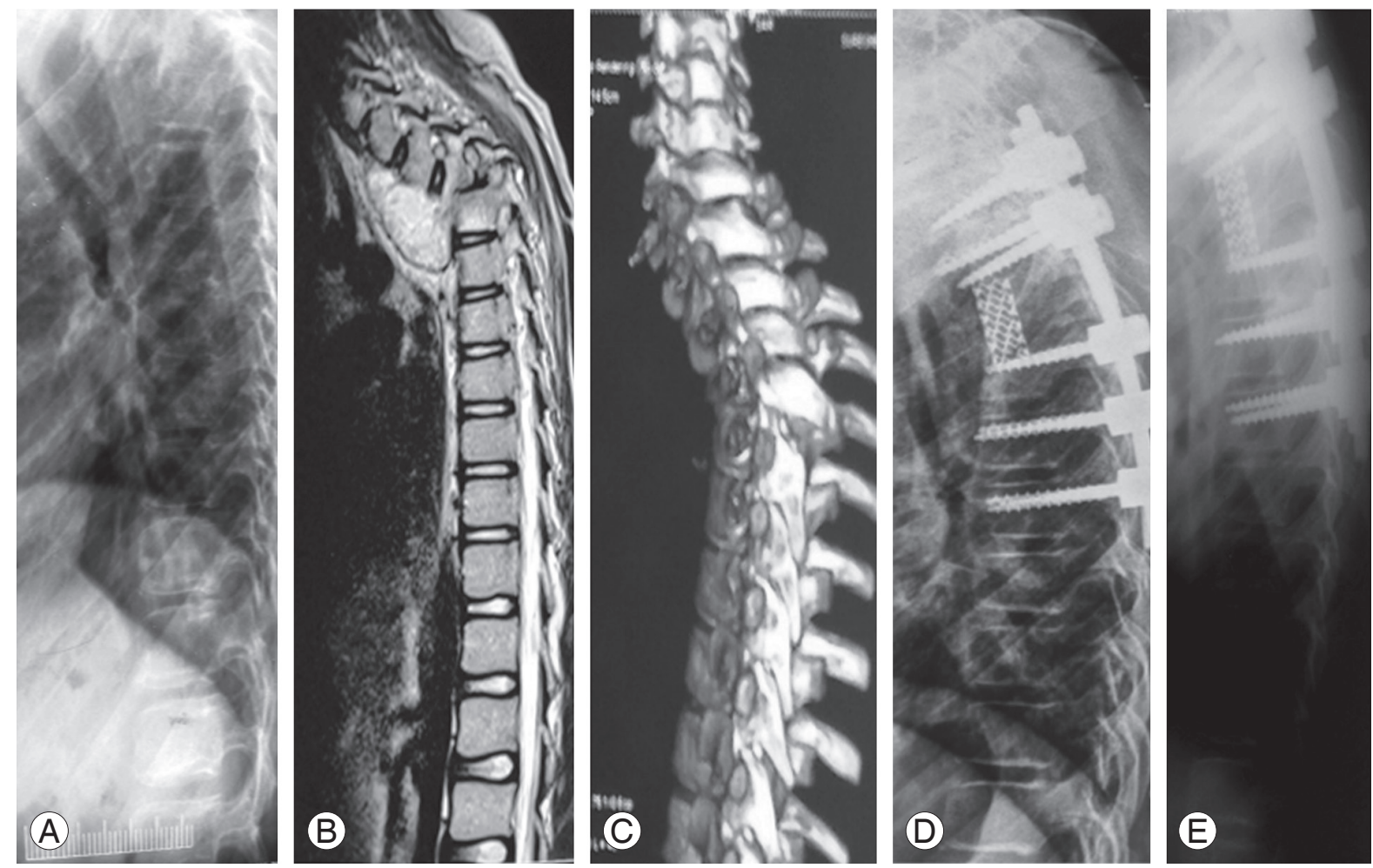

Fig. 3. A 15-year-old male with D3-D5 spinal tuberculosis with American Spinal Injury Association (ASIA) scale C classification and a $60^{\circ}$ Cobb angle preoperatively underwent surgery. The postoperative Cobb angle was $20^{\circ}$ with no improvement in neurology. At the 12 -month follow-up, the Cobb angle was $18^{\circ}$ with improvement in neurology to ASIA E. (A) Preoperative X-ray. (B) Preoperative magnetic resonance imaging. (C) Preoperative computed tomography scan. (D) Postoperative X-ray. (E) Follow-up X-ray.

paraspinal abscess $[16,17]$.

Various surgical methods have been used to treat patients with spinal TB, but few studies have been reported on the use of one-stage posterior decompression, interbody grafts and transpedicular instrumentation, and circumferential fusion for the treatment of patients with thoracolumbar spinal TB. Because the inflammation is usually located in the anterior aspect, anterior debridement and strut grafts are recommended by some surgeons [20]. This method can provide direct access to the disease focus, effective debridement of the lesion, and decompression of the spinal cord. However, the procedure may result in the progression of kyphosis as a consequence of failure of the bone graft [20-22].

In view of the complications of this method, isolated posterior instrumentation [23] or additional posterior instrumentation, which is applied to reduce kyphotic deformities and prevent correction loss and graft failure, has been applied by some surgeons [24,25]; however, this combined procedure has a longer operation time and healing duration and higher surgical trauma. We preferred the posterior approach because it reduces total operative time and morbidity and allows early mobilization, which reduces the complication risk of long-term bed rest and decreases hospitalization cost. In addition, a posterior approach offers good access to the spinal canal for efficient decompression of the neural elements, particularly in cases of epidural suppuration, and renders good clinical results [26,27].

Afterwards, a postoperative regimen of anti-TB drugs allows the drugs to effectively enter pathologic sites to kill tuberculomyces and recover the areas of bone that are infiltrated by, but not necrotic with, tuberculous disease and to resolve possible remaining abscesses that cannot be completely drained by posterior decompression [28]. Of the 20 patients, nine were already on medical management of spinal TB. The reasons for failure of medical management were a delay in the diagnosis or treatment, noncompliance of patients, involvement of more than two vertebrae, increase in the duration of the disease, decrease in the immunity of the patient to fight the disease, and unacceptable sagittal malalignment leading to pain in 

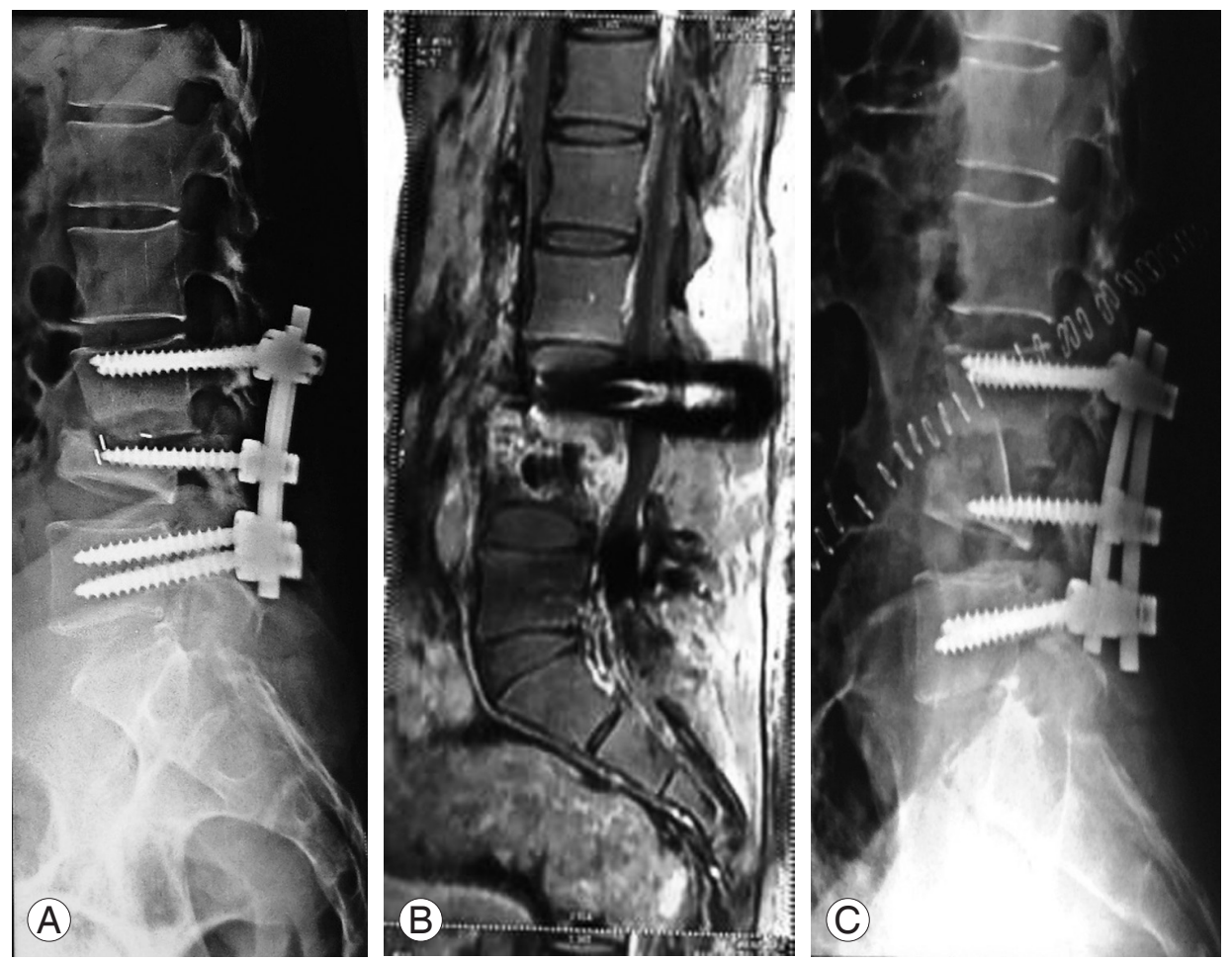

Fig. 4. Three months back operated L3-L4 spinal tuberculosis with American Spinal Injury Association E classification with complaint of persistent back pain and X-ray and magnetic resonance imaging (MRI) suggesting pseudarthrosis with localized kyphosis. The patient underwent surgery to remove a cage, and anterior tricortical strut grafting was performed using the anterior retroperitoneal approach. No worsening of neurology was observed. (A) After revision. (B) Before revision. (C) Before revision MRI.

spite of a healed lesion.

The surgical approaches are divided into posterior and anterior. Posterior approaches include transpedicular and lateral extracavitary approaches and anterior approaches include transthoracic and retroperitoneal approaches. In the present study, we performed the procedure of onestaged posterior debridement, pedicle fixation, and circumferential fusion using the transpedicular approach. In all patients, diagnosis should be confirmed by culture and sensitivity of the pathological specimen, histopathology testing, and a TB gene expert to detect sensitivity to routine drugs, according to RNTCP criteria.

The ASIA scale scores were significantly better at the final follow-up visit than those preoperatively $(p<0.05)$. The VAS scores of the patients decreased from 6.9 preoperatively to 2.55 at the final follow-up visit. The preoperative and postoperative mean kyphotic Cobb angle were $27.5^{\circ}$ and $6.25^{\circ}$ in the present study, and the difference was statistically significant $(p<0.05)$. There were no intraoperative complications. Postoperatively, two patients were diagnosed with nonunion. Refixation was performed in one patient (Fig. 2), and the removal of the cage and tricortical bone grafting by the anterior retroperitoneal approach was performed on the other patient (Fig. 4). No significant loss of deformity correction was noted in any patient at the final follow-up. It was concluded from our study that the final outcome of one-stage posterior approach was satisfactory.

Advantages of one-staged posterior surgery are kyphosis correction, single-stage procedure, low rate of infection, decreased hospital stay, and low blood loss. Disadvantages of one-staged posterior surgery include a risk of damage to the cord, thereby increasing the chances of neurological deficit, inability to use a bigger cage, difficulty in anteriorly approaching the lumbar spine as the nerve roots cannot be sacrificed, and involvement of more levels for fusion.

In conclusion, the procedure of single-staged posterior debridement, transpedicular screw fixation, and circumferential fusion by a transpedicular approach is an effective and safe procedure for surgical treatment of mono- or polysegmental thoracic and lumbar spinal TB. The antitubercular treatment protocol as per RNTCP guidelines 
is effective in ameliorating the disease and reducing the bacterial load. Despite the satisfactory results of our series, we acknowledge some limitations. First, the duration of follow-up was only long enough for short-term results but did not provide enough time for the final result. Longterm follow-up for this procedure will be necessary to further assess its application in the treatment of thoracolumbar spinal TB. Moreover, the small sample of patients limits the power of our conclusions, and a larger, randomized, controlled trial is required to elucidate the benefits and risks of our method. However, data may serve to aid decision-making and the design of future prospective studies.

\section{Conclusions}

The procedure of posterior decompression, fixation, and circumferential fusion using a transpedicular approach performed for treatment of thoracolumbar spinal TB is effective, safe, and excellent in correcting and maintaining kyphosis; however, long-term follow-up is needed.

\section{Conflict of Interest}

No potential conflict of interest relevant to this article was reported.

\section{References}

1. Tuli SM. Severe kyphotic deformity in tuberculosis of the spine. Int Orthop 1995;19:327-31.

2. Al-Sebai MW, Al-Khawashki H, Al-Arabi K, Khan F. Operative treatment of progressive deformity in spinal tuberculosis. Int Orthop 2001;25:322-5.

3. Martin NS. Pott's paraplegia: a report on 120 cases. J Bone Joint Surg Br 1971;53:596-608.

4. Guirguis AR. Pott's paraplegia. J Bone Joint Surg Br 1967;49:658-67.

5. Ito H, Tsuchiya J, Asami G. A new radical operation for Pott's disease: report of ten cases. J Bone Joint Surg Am 1934;15:499-515.

6. Rajasekaran S, Prasad Shetty A, Dheenadhayalan J, Shashidhar Reddy J, Naresh-Babu J, Kishen T. Morphological changes during growth in healed childhood spinal tuberculosis: a 15-year prospective study of 61 children treated with ambulatory chemotherapy. J Pediatr Orthop 2006;26:716-24.
7. Houshian S, Poulsen S, Riegels-Nielsen P. Bone and joint tuberculosis in Denmark: increase due to immigration. Acta Orthop Scand 2000;71:312-5.

8. Almeida A. Tuberculosis of the spine and spinal cord. Eur J Radiol 2005;55:193-201.

9. World Health Organization. Global tuberculosis control 2015 [Internet]. Geneva: World Health Organization; 2015. Available from: http://www.who.int/tb/ publications/global_report.

10. Oguz E, Sehirlioglu A, Altinmakas M, et al. A new classification and guide for surgical treatment of spinal tuberculosis. Int Orthop 2008;32:127-33.

11. Boehme CC, Nabeta P, Hillemann D, et al. Rapid molecular detection of tuberculosis and rifampin resistance. N Engl J Med 2010;363:1005-15.

12. Central TB Devision, Ministry of Health and Family Welfare. TB India 2010: RNTCP annual status report. New Delhi: Ministry of Health and Family Welfare; 2010.

13. World Health Organization. Treatment of tuberculosis: guidelines. 4th ed. Geneva: World Health Organization; 2010.

14. Lenke LG, Betz RR, Harms J, et al. Adolescent idiopathic scoliosis: a new classification to determine extent of spinal arthrodesis. J Bone Joint Surg Am 2001; 83A:1169-81.

15. Lonnroth K, Raviglione M. Global epidemiology of tuberculosis: prospects for control. Semin Respir Crit Care Med 2008;29:481-91.

16. Jain AK. Tuberculosis of the spine. Clin Orthop Relat Res 2007;460:2-3.

17. Tuli SM. Tuberculosis of the spine: a historical review. Clin Orthop Relat Res 2007;460:29-38.

18. Rezai AR, Woo HH, Errico TJ, Cooper PR. Contemporary management of spinal osteomyelitis. Neurosurgery 1999;44:1018-25.

19. Przybylski GJ, Sharan AD. Single-stage autogenous bone grafting and internal fixation in the surgical management of pyogenic discitis and vertebral osteomyelitis. J Neurosurg 2001;94(1 Suppl):1-7.

20. Bailey HL, Gabriel M, Hodgson AR, Shin JS. Tuberculosis of the spine in children. Operative findings and results in one hundred consecutive patients treated by removal of the lesion and anterior grafting. J Bone Joint Surg Am 1972;54:1633-57.

21. Fountain SS, Hsu LC, Yau AC, Hodgson AR. Progressive kyphosis following solid anterior spine fusion in 
children with tuberculosis of the spine: a long-term study. J Bone Joint Surg Am 1975;57:1104-7.

22. Upadhyay SS, Sell P, Saji MJ, Sell B, Yau AC, Leong JC. 17-year prospective study of surgical management of spinal tuberculosis in children. Hong Kong operation compared with debridement surgery for short- and long-term outcome of deformity. Spine (Phila Pa 1976) 1993;18:1704-11.

23. Shen $X$, Huang $X, X i a o ~ S$, et al. Surgical treatment of selected patients with multilevel contiguous thoracolumbar spinal tuberculosis by only posterior instrumentation without any bone fusion. Int J Clin Exp Med 2015;8:18611-9.

24. Sundararaj GD, Behera S, Ravi V, Venkatesh K, Cherian VM, Lee V. Role of posterior stabilisation in the management of tuberculosis of the dorsal and lumbar spine. J Bone Joint Surg Br 2003;85:100-6.

25. Talu U, Gogus A, Ozturk C, Hamzaoglu A, Domanic
$\mathrm{U}$. The role of posterior instrumentation and fusion after anterior radical debridement and fusion in the surgical treatment of spinal tuberculosis: experience of 127 cases. J Spinal Disord Tech 2006;19:554-9.

26. Guzey FK, Emel E, Bas NS, et al. Thoracic and lumbar tuberculous spondylitis treated by posterior debridement, graft placement, and instrumentation: a retrospective analysis in 19 cases. J Neurosurg Spine 2005;3:450-8.

27. Malawski S. Clinical value of the posterolateral approach, supplemented with pedicle excision of the arch, to the anterior wall of the spinal canal within the thoracolumbar (Th11-L1) and lumbar spine. Chir Narzadow Ruchu Ortop Pol 1994;59:265-73.

28. Nene A, Bhojraj S. Results of nonsurgical treatment of thoracic spinal tuberculosis in adults. Spine J 2005;5:79-84. 